\title{
Spontaneous Rupture of a Giant Diaphragmatic Hydatid Cyst into the Intrapleural Space
}

\author{
Erkan Dervisoglu ${ }^{a}$ Salih Topcu ${ }^{b}$ Serife Tuba Liman ${ }^{b} \quad$ Ahmet Yilmaz $^{a}$ \\ Departments of ${ }^{a}$ Internal Medicine (Division of Nephrology) and ${ }^{b}$ Thoracic Surgery, Faculty of Medicine, \\ Kocaeli University, Kocaeli, Turkey
}

\section{Key Words}

Hydatid disease - Diaphragm $\cdot$ Hydatid cyst rupture Multislice computed tomography

\begin{abstract}
Objective: We report a case of giant diaphragmatic hydatid cyst which ruptured spontaneously into the intrapleural space in a patient with coexistent giant hepatic hydatid cyst. Clinical Presentation and Intervention: A 62-year-old female was admitted for dyspnea, nausea, vomiting, and right thoracic pain. Clinical findings, laboratory and radiological examinations including multislice computed tomography scan were consistent with the diagnosis of a giant diaphragmatic hydatid cyst which ruptured into the intrapleural space. Surgical intervention was performed through thoracotomy and phrenotomy in a one-stage operation for both cysts. Conclusion: This case shows that hydatid cysts of the diaphragm can rupture into the intrapleural space spontaneously. One-stage operation through thoracotomy may be successful for the surgical intervention for diaphragmatic hydatid cysts with coexistent hepatic cyst.
\end{abstract}

(C) 2007 S. Karger AG, Basel

1011-7571/08/0171-0086\$24.50/0

Fax +4161306 1234

E-Mail karger@karger.ch

www.karger.com
Accessible online at: www.karger.com/mpp

\section{Introduction}

Hydatid disease or echinococcosis is a worldwide parasitic zoonosis. The eggs of the tapeworm Echinococcus granulosus excreted by carnivores may infect various species of natural intermediate host animals such as sheep, cattle, deer and accidentally humans. Hydatid cysts of $E$. granulosus primarily form in the liver or in the lungs, but may rarely be found in different organs [1]. The parasite can settle in any organ or tissue in the human body but diaphragmatic localization is rare with the incidence of only about $1 \%$ [2]. Rupture of the hydatid cyst may be spontaneous, iatrogenic or traumatic [3]. Spontaneous rupture of a hydatid cyst in the diaphragmatic localization is unusual. We report a case of a giant diaphragmatic hydatid cyst which ruptured spontaneously into the right intrapleural space in a patient with a coexistent giant hepatic hydatid cyst.

\section{Case Report}

A 62-year-old woman was admitted with dyspnea, nausea, vomiting, and right-sided chest pain for 1 month. On physical examination her blood pressure was 100/60 $\mathrm{mm} \mathrm{Hg}$, heart rate 82 bpm and body temperature $37.2^{\circ} \mathrm{C}$. The patient's breath sounds were decreased at the mid-base of the right chest and $6 \mathrm{~cm}$ hepatomegaly was detected. Blood work results were as follows: $\mathrm{Hb} 11.1$ 
$\mathrm{g} / \mathrm{dl}, \mathrm{WBC} 17,100 / \mathrm{mm}^{3}$, erythrocyte sedimentation rate $110 \mathrm{~mm} / \mathrm{h}$, C-reactive protein level $26.4 \mathrm{mg} / \mathrm{dl}$ (normal: $<0.8 \mathrm{mg} / \mathrm{dl}$ ). Serum biochemistry work-up revealed a serum creatinine of $2.6 \mathrm{mg} / \mathrm{dl}$ (ref. 0.7-1.3), blood urea nitrogen of $51 \mathrm{mg} / \mathrm{dl}$ (ref. 7-25), and albumin of $2.9 \mathrm{~g} / \mathrm{dl}$. Serum bilirubin and liver enzymes were within normal range. Urinalysis showed a specific gravity of 1.023 and was positive for protein. Renal ultrasound showed normal-size kidneys, non-distended urinary bladder and no evidence of hydronephrosis. Acute renal failure due to sepsis was suspected. The patient was hospitalized. After placement of a dual-lumen hemodialysis (HD) catheter into the right internal jugular vein, HD treatment was started on hospital day 1 . Chest radiography revealed right-sided pleural effusion (fig. 1). A chest tube was inserted in the right hemithorax and $500 \mathrm{ml}$ of purulent material were drained. The microbiological examination of the fluid revealed leukocytes with Gramnegative bacilli and Gram-positive cocci. A right-sided empyema was diagnosed and cefoperazone/sulbactam was started at a daily dose of $2 \mathrm{~g}$. Cultures of the fluid remained negative. HD therapy was continued for the subsequent 4 days. Her gastrointestinal symptoms improved. Thoracoabdominal multislice computed tomography (CT) showed a mass lesion with mixed solid and cystic components in the diaphragm and a second lesion in right lobe of the liver, consistent with hydatid cysts (fig. 2a, b). Indirect hemagglutination (IHA) test was positive for hydatid disease. Oral albendazole at a daily dose of $15 \mathrm{mg} / \mathrm{kg}$ was started as medical therapy.

Surgery was performed on the 7th day of hospitalization. Following a right thoracotomy through the seventh intercostal space, multiple daughter vesicles were observed in the intrapleural space and the mediastinum. The lesions were removed. The intrapleural space was irrigated with $1 \%$ povidone-iodine solution. No cyst was seen in the parenchyma of the lung. A hydatid cyst originating from the diaphragm was observed. The cyst was surrounded by the layers of diaphragm. Cystotomy was performed and daughter vesicles were removed from the cyst. The germinative membrane was excised. The giant hepatic cyst was then reached through a subsequent transdiaphragmatic incision on the anterolateral side of the diaphragm and cystic fluid including daughter vesicles was aspirated. After excision of the germinative membrane a Pezzer drain was inserted into the cavity and the cystic cavity of the liver was capitonnaged (folding of the pericystic zone by sutures). The cystic cavity of the diaphragm was also capitonnaged. After insertion of two chest tubes, the thoracotomy incision was closed.

Cefoperazone/sulbactam was continued for 21 days. Four weeks after admission, the patient was taken off renal replacement therapy. Serum creatinine decreased to $1.1 \mathrm{mg} / \mathrm{dl}$ on hospital day 40. The amount of drained material gradually decreased but never stopped completely. The patient was discharged on hospital day 56 with a prescription for oral albendazole, and with two chest tubes with Heimlich valves which were to be removed when the drainage stopped.

\section{Discussion}

Hydatid disease presents as hydatid cysts primarily in the liver and lungs. Although cysts may be asymptomatic for many years, they may become clinically manifest

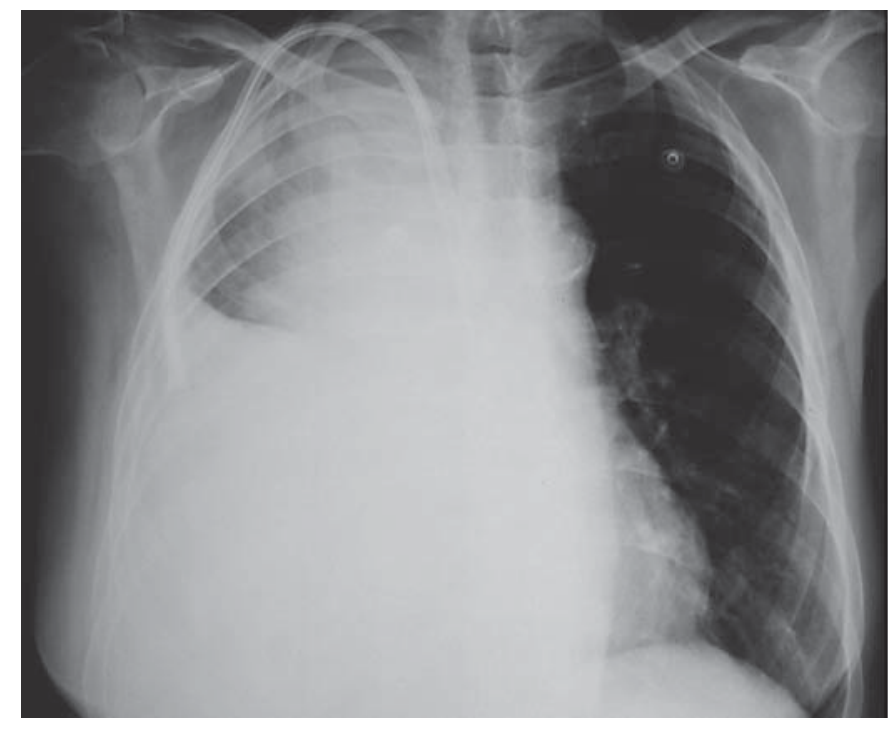

Fig. 1. Chest radiography shows right-sided pleural effusion and increased density at the upper and mid zones of the right lung. A dual lumen hemodialysis catheter in the superior vena cava is also seen.
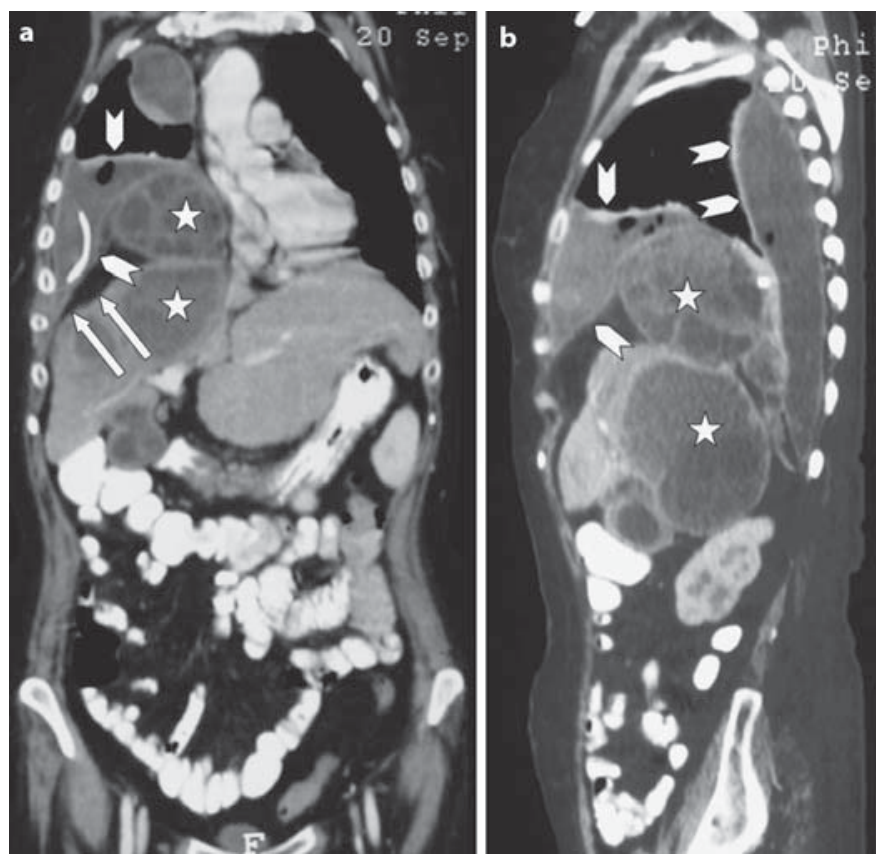

Fig. 2. Thoracoabdominal multislice CT images (a coronal plane, b sagittal plane) demonstrate mass lesions with mixed solid and cystic components located in the diaphragm $(11 \times 10 \times 9 \mathrm{~cm})$ and right lobe of the liver $(13 \times 11 \times 10 \mathrm{~cm})$ consistent with a hydatid cyst (stars). Pleural effusion within the major fissure and posterior pleural space is seen (arrowheads). The diaphragm is shown with arrows. A chest tube is also seen in the intrapleural space. 
due to expansion, rupture, and/or pyogenic infection [1, $4,5]$. Our case shows that hydatid cysts of the diaphragm can rupture into the intrapleural area spontaneously. Diaphragmatic involvement of hydatid disease is rare and most of the previously reported cases are associated with liver hydatid cysts [2]. In our case, the diaphragmatic cyst was separate from the coexisting liver cyst.

Spontaneous rupture of a diaphragmatic hydatid cyst in this localization is a very rare entity. To our knowledge there is only one report regarding the spontaneous rupture of a hydatid cyst of the diaphragm [6]. In that patient, after surgical intervention via thoracotomy, the outcome was reported to be good except for local relapses twice, in the second and fourth years of follow-up. However, medical treatment with albendazole was not reported for the patient. Since medical treatment with albendazole or mebendazole is recommended postoperatively in patients with perforated cysts [7], we administered albendazole for 6 months. In addition, the patient was asked to return for chest radiography and abdominal ultrasonography at 6-month intervals to detect relapses.

The clinical presentation of hydatid disease depends on the size and site of the cyst. Preoperative diagnosis of hydatid cysts may be made by ultrasonography and confirmed by CT [8]. On CT, it can be easily differentiated from other mass lesions due to its typical three-layered structure and the ring-like polycyclic calcification [9]. A variety of serological tests are available for the diagnosis, screening and postoperative follow up for recurrence. These are hydatid immunoelectrophoresis, enzyme- linked immunosorbent assay, latex agglutination and IHA test [8]. In our case the diagnosis was established with a multislice CT scan. Furthermore, the IHA test was found to be positive for hydatid disease.

Surgical intervention should be the treatment of choice for hydatid disease. In diaphragmatic hydatid cysts total excision of the cyst via thoracotomy is a suitable approach [2]. Access through a thoracic approach, which implies right thoracotomy with phrenotomy, has been effective in the simultaneous management of concomitant right-sided pulmonary and hepatic hydatid cysts [10]. If a diaphragmatic hydatid cyst is associated with a liver cyst, or an independent liver cyst exists along with a diaphragmatic cyst as in our case, these cysts can be treated simultaneously in a one-stage operation.

\section{Conclusion}

This case demonstrates that hydatid cysts of the diaphragm can rupture into the intrapleural space spontaneously. One-stage operation through thoracotomy may be successful for the surgical intervention for diaphragmatic hydatid cysts with coexistent hepatic cysts.

\section{Acknowledgement}

We would like to especially thank Gür Akansel, MD, Department of Radiology at Kocaeli University, Faculty of Medicine, for his valuable contributions.

\section{References}

1 Kilimcioglu AA, Ozkol M, Bayindir P, Girginkardesler N, Ostan I, Ok UZ: The value of ultrasonography alone in screening surveys of cystic echinococcosis in children in Turkey. Parasitol Int 2006;55:273-275.

-2 Eren S, Ulku R, Tanrikulu AC, Eren MN: Primary giant hydatid cyst of the diaphragm. Ann Thorac Cardiovasc Surg 2004;10:118119.

3 Sahin E, Kaptanoglu M, Nadir A, Ceran C: Traumatic rupture of a pulmonary hydatid cyst: a case report. Ulus Travma Acil Cerrahi Derg 2006;12:71-75.
4 Qian ZX: Thoracic hydatid cysts: a report of 842 cases treated over a thirty-year period. Ann Thorac Surg 1988;46:342-346.

$\checkmark 5$ Kurul IC, Topcu S, Altinok T, Yazici U, Tastepe I, Kaya S, Cetin G: One-stage operation for hydatid disease of lung and liver: principles of treatment. J Thorac Cardiovasc Surg 2002;124:1212-1215.

-6 Daali M, Hssaida R: Rupture of a hydatid cyst of the diaphragm in the pleura: a case report. Ann Chir 2000;125:493-495.

7 Ulku R, Eren N, Cakir O, Balci A, Onat S: Extrapulmonary intrathoracic hydatid cysts. Can J Surg 2004;47:95-98.
8 Abu-Eshy SA: Some rare presentations of hydatid cyst (Echinococcus granulosus). J R Coll Surg Edinb 1998;43:347-352.

\9 Kotoulas C, Foroulis C, Letsas K, Kostikas K, Konstantinou M: Bilious pericardial effusion at initial presentation in a patient with lung cancer. World J Surg Oncol 2003;1:24.

- 10 Topcu S, Kurul IC, Altinok T, Yazici U, Demir A: Giant hydatid cysts of lung and liver. Ann Thorac Surg 2003;75:292-294. 